\title{
Guidelines
}

European Thyroid Journal
Eur Thyroid J 2019;8:227-245

DOI: $10.1159 / 000502229$
Received: May 21, 2019

Accepted after revision: July 19, 2019 Published online: August 28, 2019

\section{European Thyroid Association Guidelines for the Treatment and Follow-Up of Advanced Radioiodine-Refractory Thyroid Cancer}

\author{
Laura Fugazzola ${ }^{a, b}$ Rossella Elisei ${ }^{c}$ Dagmar Fuhrer ${ }^{d}$ Barbara Jarzab $^{\mathrm{e}}$ \\ Sophie Leboulleux ${ }^{f}$ Kate Newbold ${ }^{g}$ Jan Smit ${ }^{\text {h }}$ \\ ${ }^{a}$ Division of Endocrine and Metabolic Diseases, IRCCS Istituto Auxologico Italiano, Milan, Italy; ${ }^{b}$ Department of \\ Pathophysiology and Transplantation, University of Milan, Milan, Italy; ${ }^{C}$ Unit of Endocrinology, Department of \\ Clinical and Experimental Medicine, University of Pisa, Pisa, Italy; ${ }^{d}$ Department of Endocrinology, Diabetes and \\ Metabolism, Endocrine Tumour Center at West German Cancer Center, University Hospital Essen, University \\ of Duisburg-Essen, Duisburg, Germany; ${ }^{e}$ Department of Nuclear Medicine and Endocrine Oncology, Maria \\ Sklodowska-Curie Institute, Oncology Center, Gliwice Branch, Gliwice, Poland; ${ }^{f}$ Department of Nuclear Medicine \\ and Endocrine Oncology, Gustave Roussy and Université Paris Saclay, Villejuif, France; ${ }^{9}$ Royal Marsden NHS \\ Foundation Trust and Institute of Cancer Research, London, UK; ${ }^{h}$ Division of Endocrinology, Department of Internal \\ Medicine, Radboud University Medical Center, Nijmegen, The Netherlands
}

\section{Keywords}

European Thyroid Association - Advanced thyroid cancer . Radioiodine refractory thyroid cancer - Multikinase inhibitors · Local treatments · Lenvatinib · Sorafenib · Familial counselling $\cdot$ Rehabilitation

\footnotetext{
Abstract

The vast majority of thyroid cancers of follicular origin (TC) have a very favourable outcome, but $5-10 \%$ of cases will develop metastatic disease. Around $60-70 \%$ of this subset, hence less than $5 \%$ of all patients with TC, will become radioiodine refractory (RAI-R), with a significant negative impact on prognosis and a mean life expectancy of 3-5 years. Since no European expert consensus or guidance for this challenging condition is currently available, a task force of TC experts was nominated by the European Thyroid Association (ETA) to prepare this document based on the principles of clinical evidence. The task force started to work in September 2018
}

and after several revision rounds, prepared a list of recommendations to support the treatment and follow-up of patients with advanced TC. Criteria for advanced RAI-R TC were proposed, and the most appropriate diagnostic tools and the local, systemic and palliative treatments are described. Systemic therapy with multikinase inhibitors is fully discussed, including recommendations on how to start it and at which dosage, on the duration of treatment, and on the management of side effects. The appropriate relationship between the specialist and the patient/family as well as ethical issues are covered. Based on the available studies and on personal experience, the experts provided 39 recommendations aimed to improve the management of advanced RAI-R TCs. Above all of them is the indication to treat and follow these patients in a specialized setting which allows the interaction between several specialists in a multidisciplinary team. (c) 2019 European Thyroid Association Published by S. Karger AG, Basel

\section{KARGER}

E-Mail karger@karger.com www.karger.com/etj (c) 2019 European Thyroid Association

Published by S. Karger AG, Basel
Laura Fugazzola

Division of Endocrine and Metabolic Diseases, IRCCS Istituto Auxologico Italiano Department of Pathophysiology and Transplantation, Università degli Studi di Milano P. le Brescia 20, IT-20149, Milan (Italy)

E-Mail laura.fugazzola@unimi.it 


\section{Premise}

Few prospective studies are available on the management of patients with advanced thyroid cancer (TC), and the decision making is mostly based on the personal experience of physicians. The present guidelines are mainly based on the knowledge derived from clinical practice of a group of ETA (European Thyroid Association) experts who regularly diagnose, treat, and follow up the most aggressive forms of TC.

\section{Epidemiology and Classification of TC}

According to the European Network of Cancer Registries (https://www.encr.eu, January 2017), in Europe an estimated 53,000 TC cases were newly diagnosed in 2012. In the same year, 6,300 Europeans are estimated to have died of TC. Women have a 3-fold higher estimated age-standardized rate of TC incidence (IRR) than men, with 9.3 and 3.1 cases per 100,000 person-years, respectively (mean 6.3 cases). The same incidences are recorded in all developed countries, though in Europe there are wide regional differences. In particular, the highest estimated age-standardized IRR are recorded in Lithuania (15.5 cases per 100,000 person-years), Italy (13.5), Austria (12.4), Croatia (11.4), and Luxembourg (11.1). The 5 -year relative survival of TC in Europe ranges from 80 to $90 \%$. TC includes histological types with extremely different prevalence. Among tumours originating from the follicular epithelium, differentiated TC (DTC) is the most common type and includes the papillary and follicular histotypes, making up about $85-95 \%$ of all TCs, whereas Hürtle cell carcinomas (HC) and poorly differentiated TC account for 2-5\% each. Anaplastic thyroid carcinoma comprises approximately $1.7 \%$ of all cases of $\mathrm{TC}$ and is a very aggressive malignancy with an average survival time following diagnosis of 6 months or less. Finally, medullary TC, originating from the parafollicular C cells of neuroendocrine origin, accounts for 3-5\% of all TCs. It can be classified as sporadic (75\% of cases), or familial MTC. Familial forms are generally bilateral with multiple foci, are due to germline mutations in the RET gene and can associate with other diseases in the context of Multiple Endocrine Neoplasias, MEN 2A, and MEN 2B.

These guidelines focus on radioiodine refractory (RAIR) TCs; hence, only TCs originating from the follicular epithelium and predicted to be capable of radioiodine uptake, will be discussed.
Among DTCs, papillary TC (PTC) is the most common type, and it is defined as a malignant epithelial tumour with evidence of follicular differentiation and a series of specific nuclear features. According to the last WHO classification [1], it can be sub-classified according to the pathological features as papillary microcarcinoma, as encapsulated, follicular, diffuse sclerotizing, tall cell, columnar cell, cribriform-morular, and hobnail cell variants, and as fibromatosis/fasciitis stroma, solid/trabecular, oncocytic, fusiform cell, clear cell and Warthin type variants. Follicular TC (FTC) is defined as a malignant tumour lacking the nuclear characteristics of PTC. Three main subtypes are established: (1) minimally invasive FTC (encapsulated and with invasion only of the capsule); (2) encapsulated angioinvasive FTC; and (3) widely invasive FTC. In the new classification of the WHO, HC or oncocytic tumours are regarded as a separate group. The $\mathrm{HC}$ carcinomas are divided into minimally invasive, encapsulated angioinvasive, and widely invasive subtypes, following the same criteria as those applied to FTC. Poorly differentiated thyroid carcinoma (PDTC) is a follicular neoplasm that shows limited evidence of follicular cell differentiation, which lies morphologically and behaviourally between differentiated (follicular and papillary) and anaplastic carcinomas [1]. The histopathological diagnostic criteria for PDTC are defined in the Turin Consensus proposal: (1) malignant thyroid follicular cell neoplasm (presence of invasion and/or metastasis); (2) solid, trabecular, or insular growth pattern; (3) absence of the conventional nuclear features of PTC; (4) at least one of the following three features: convoluted nuclei (defined as a small round hyperchromatic nuclei with convolutions of the nuclear membrane), 3 or more than 3 mitoses per 10 high-power fields, and tumour necrosis [2]. Vascular invasion, lymph node metastases, extrathyroidal tumour extension are very common in PDTC, and distant metastases may be detected at presentation or shortly after initial diagnosis $[3,4]$. PDTCs are frequently resistant to radioactive iodine, and have a 5 -year overall survival rate of $60-85 \%$ [5]. PDTC with predominant oncocytic features defines the oncocytic variant of PDTC, which is characterized by an even poorer prognosis in terms of overall and tumourspecific survival [6].

DTCs, HCs, or PDTCs which, during tumour progression, lose their ability to uptake and concentrate radioiodine, and in some cases to produce thyroglobulin ( $\mathrm{Tg}$ ), are commonly clinically defined as dedifferentiated TCs (DeDTC). DeDTC is not a histopathological entity and can be thus diagnosed only during the course of the dis- 
ease. Indeed, current WHO classification does not precisely define the pathological criteria of dedifferentiation, though pathologists refer to this term when they observe signs of dedifferentiation in nuclei, in the growth pattern, a high mitotic activity, and necrosis in the recurrent or metastatic lesion of a patient with primary diagnosis of DTC $[5,7,8]$.

The molecular pattern of DTCs is often characterized by mutations in the MAPK and PI3K pathways, prevalently BRAF and RAS mutations in PTC and RAS and PAX8/PPAR $\gamma$ mutations in FTC [9]. During the dedifferentiation process, DTCs acquire subsequent mutations, and they frequently harbour, as PDTC, multiple somatic genetic alterations including TERT promoter and EIF1AX mutations [10, 11].

In terms of mortality, the IRR are 0.7 and 0.5 cases per 100,000 person-years for women and men, respectively (mean 0.6 deaths). The vast majority of TCs have an excellent prognosis, but $5-10 \%$ of cases (6-7 new cases/ year/million) will develop metastatic disease, mostly located in lungs and bones. The prognosis of these metastatic cases remains favourable as long as they maintain a response to the treatment with radioiodine. Unfortunately, around $60-70 \%$ of this subset, still less than $5 \%$ of all patients with TC, will become RAI-R, representing 4-5 new cases/year/million. This has a significant impact on prognosis, with the 10-year survival rate dropping to less than $20 \%$ and the mean life expectancy to $3-5$ years once the disease is RAI-R [12].

\section{Recommendation}

[R1] The development of dedifferentiation and RAI refractoriness during the course of the DTC should be taken into consideration.

[R2] Poorly differentiated TC is frequently RAI-R and harbours a high risk of recurrent or metastatic disease and cancerrelated death.

[R3] RAI refractoriness occurs in around $60-70 \%$ of metastatic TCs, but less than $5 \%$ of all patients with TC.

\section{Definition of RAI-R TC}

In recent years, many new drugs have been investigated for the treatment of progressive RAI-R TC. Currently, 2 multikinase inhibitors (MKI) have been approved for treatment by the Food and Drug Administration (FDA) and the European Medicines Agency (EMA), e.g. sorafenib and lenvatinib. The efficacy of both drugs

2019 ETA Guidelines for the Treatment of Advanced RAI-R TC has been demonstrated in phase III studies (DECISION study for sorafenib [13] and SELECT study for lenvatinib [14] and has been confirmed in "real-life" studies [1519].

Although criteria for prescribing both MKIs include RAI-R disease and progression, the definition of both categories can be challenging in clinical practice. In international guidelines [20] and scientific publications $[14,20-30]$, there is no consensus on the definition of RAI-R disease. In most publications, the following categories are proposed: (1) the absence of uptake of RAI in all lesions on scintigraphy; (2) the absence of RAI uptake in some but not all lesions; (3) progression despite uptake of RAI; (4) reaching the maximum recommended activity of RAI.

\section{Absence of Uptake of RAI on Scintigraphy}

Publications differ in their criteria for absence of RAI uptake on scintigraphy, some stating post-RAI therapy scintigraphy $[13,14,20]$, others diagnostic scintigraphy or both $[25,27]$. It should be noted that diagnostic scintigraphy, even with I-124, is inferior to post-therapeutic scintigraphy [31]. In addition, factors that influence RAI uptake, including patient preparation, administered activity, and scanning protocols are often not specified, though these factors may influence RAI accumulation by the tumour and the sensitivity of detection [28]. In contrast, the presence of RAI uptake does not guarantee RAIsensitive disease $[32,33]$.

\section{Absence of RAI Uptake in Some but Not All Lesions}

Although a mixed pattern with some lesions accumulating RAI and others not is also considered RAI refractory disease in most publications, it is uncertain whether this is correct, as no study has shown that continuation of RAI treatment in this situation is not beneficial. A combination of RAI therapy for RAI-avid lesions and local treatment for one or a limited number of RAI refractory lesions may be considered in certain patients.

\section{Progression despite Uptake of RAI}

Although most publications agree that progression is a criterion for RAI-R disease, huge variation exists in the definition of progression. Radiological progression is widely accepted as an important criterion, but some studies also include biochemical progression [20, 25, 27]. Metrics for radiological progression are not specified in most publications. In clinical trials, RECIST 1.1 criteria [34] are strictly followed to define progression. Nevertheless, it should be noted that these criteria are not designed 
for individual patient care since progression, as defined by RECIST, is not identical to clinically relevant progression. As an example, the appearance of a small new lesion is technically defined as radiological progression, but can be clinically irrelevant. On the other hand, even a minor increase in volume can have a major clinical impact depending on the anatomical position of the metastatic lesion. A second uncertainty in defining progression is progression rate. In most publications, there is no time frame indicated concerning progression. In the DECISION and SELECT trials, time periods were defined as 14 and 12 months, respectively $[12,13]$, some publications mention intervals of 6-12 months, whereas others do not specify time frames.

\section{Reaching the Maximum Activity of RAI}

In a study from France, it was observed that after a cumulative activity of $22.2 \mathrm{GBq}$, no further complete remissions were achieved in patients with metastasized TC [12]. Hence, it was concluded that further RAI treatment exceeding $22 \mathrm{GBq}$ would not be beneficial. However, this conclusion, based on one study, cannot be translated into a universal cut-off value for RAI activity. For instance, if a patient has stable disease after multiple therapies with RAI, this should not be regarded as RAI$\mathrm{R}$ disease, as is acknowledged in the ATA guidelines [20].

\section{Recommendation}

[R4] In patients with radiologically proven lesions, RAI-R disease is almost certain in the absence of RAI uptake on SPECT-CT, performed after high-activity RAI and prepared with high TSH and a diet with low iodine content.

[R5] The question of whether to continue with RAI therapy or not in a patient without RAI uptake, or with a mixed pattern of uptake, or progressing despite RAI uptake, can only be addressed in a multidisciplinary team (MDT), weighing the benefits and disadvantages of continuing RAI eventually combined with local treatments.

[R6] The question of whether RAI therapy should be continued in patients who have received multiple treatments with RAI can only be addressed in an MDT, weighing the benefits and disadvantages of continuing RAI.

[R7] Progression should be defined as radiological progression, according to RECIST 1.1 criteria, within a clinically relevant time frame, which is usually considered 6-12 months. Tg can be used as an indicator for progression, but not as a surrogate for radiological assessment. The question whether progression is clinically meaningful cannot be answered from the literature and should be addressed in an MDT.

\section{Diagnostic Tools}

Diagnostics in advanced TC are directed to assess tumour biology and tumour burden. Clinical examination will clarify to what extent the patient is affected by local and distant tumour manifestations, e.g. tracheal and/or oesophageal compression, metastases to bone, lung, and brain. It should also include assessment of general symptoms such as pain, fatigue, changes in weight and appetite, and importantly the impact of disease on daily life. The latter is critical in relation to treatment with MKIs since side effects may significantly impact daily activities in an otherwise asymptomatic patient.

Tumour biology comprises the histological and molecular features of TC, its functional properties, in particular the ability to concentrate iodine in a therapeutically relevant manner, and the rate of tumour growth. The contribution of pathology to determining tumour biology resides on two principles: first, a careful review of the initial histopathology and, if required, a second assessment by a thyroid reference pathologist may both help to understand the aggressive disease course observed. Second, molecular pathology, in particular screening for BRAF mutations and ALK, NTRK, RET rearrangements may have a therapeutic impact, e.g. for considering targeted treatment. Furthermore, while several studies suggest that the presence of a TERT promoter mutation (or Tp53 mutation) in TC is associated with a worse prognosis and hence a higher rate of these mutations can be expected in advanced TC $[35,36]$, there is no evidence that presence of either mutation should alter or determine management. Clearly, incorporating molecular pathology into treatment decisions is a hot topic for research activities, that also includes pharmacological approaches aimed at restoring therapeutically useful 131-I uptake and lately immunotherapy [37]. In this context, genetic analyses should be directed to the evaluation of alterations targeted by drugs either approved or on trial.

The importance of high-resolution imaging and the lack of absolute criteria to define a TC as RAI-R have recently been discussed in the "Martinique principles" - a joint statement by EANM ATA, ETA, and SNMI [30]. Until better evidence is available from study data, the criteria discussed above for a likely RAI-R TC may be used (see the section Definition of RAI-R TC).

Tumour growth is assessed by sequential CT scanning, most commonly at 6- to 12-month intervals and applying RECIST 1.1 criteria to determine disease status. Recently, Tuttle and co-workers $[38,39]$ proposed that 
changes in lesion volume, e.g. in lung metastases, may be a more reliable prognostic marker for tumour growth than RECIST and may possibly facilitate the decision when to start MKI therapy. In addition to morphological imaging, important prognostic indicators may be derived from FDG-PET/CT. In a pilot study, TC patients with FDG-PET-positive tumour lesions had a significantly shorter survival than patients with metastatic TC, but no increased glucose uptake [40]. Furthermore, by comparison with RAI avidity, FDG positivity had a more profound negative impact on survival, as confirmed in an independent second study [41].

Tumour burden describes the total tumour mass in the body. Its assessment should be based on imaging including high-resolution neck ultrasonography, or CT scanning of neck, chest, abdomen, and brain, in addition to assessment for bone metastases by scintigraphy and CT. Alternatively and due to its prognostic impact, FDG$\mathrm{PET} / \mathrm{CT}$ scanning should be considered in RAI-R disease. Moreover, focused diagnostics, e.g. bronchoscopy or oesophagoduodenoscopy, may be indicated to clarify local tumour extension with current, imminent, or likely complications. Tumour burden can be roughly estimated by $\mathrm{Tg}$ levels, as well as the rapid progression and poor prognosis of the disease can be predicted by a Tg doubling time $<1$ year [42]. However, in some aggressive TC, with a poor level of Tg secretion, Tg level might underestimate tumour burden. Furthermore, in the presence of $\mathrm{TgAb}, \mathrm{Tg}$ cannot be used as a reliable tumour marker. It is worth noting that in patients with advanced TC, rhTSH test is not performed since data on the Tg-stimulated levels do not add any additional relevant information for the management of these patients.

All patients with advanced TCs require control of their thyroid function in order to maintain suppressed TSH levels. Thus, TSH and fT4 serum concentrations should be obtained at 6- to 12-month intervals and more frequently if MKI treatment is started. In case of postoperative hypoparathyroidism, regular control of calcium status is required following the recent recommendations of the American Thyroid Association [43].

Importantly, while all of the discussed diagnostic approaches and their outcomes are linked, none of them provides a "one and only" information; hence, a combination of diagnostic tools and their interpretation by physicians experienced in TC care is required to unmask the true nature of advanced TC and to enhance patientoriented decision making.

\section{Recommendation}

[R8] A careful clinical assessment is recommended at every follow-up visit. Particular attention should be paid to clinical manifestations of advanced disease and quality of life.

[R9] Laboratory assessment should include TSH, fT4, and calcium status in case of postoperative hypoparathyroidism. Tg and $\mathrm{Tg}$ antibody titre should be assessed using the locally available $\mathrm{Tg}$ assay and relying on dynamics in sequential measurements rather than absolute Tg concentrations. A change in assays should be avoided.

[R10] In advanced TC, serial imaging at 6- to 12-month intervals, e.g. with whole-body CT and applying RECIST, is useful to assess dynamics of tumour growth. 18-FDG-PET/CT scanning may yield additional information on tumour biology with a negative prognostic impact for lesions with increased glucose uptake.

[R11] No single imaging modality provides "one and all" information on tumour biology; hence, a combination of imaging techniques and where indicated, other diagnostics, e.g. endoscopy, are required to unravel which tumour lesions require local or systemic intervention.

[R12] Histopathology together with molecular pathology may be predictive of the course of TC, but so far has limited impact on management. However, molecular pathology may be useful for individualized, off-label targeted therapies, when no clinical studies are available.

[R13] Interpretation of all diagnostics investigations that contribute to the assessment of advanced TC biology requires a multidisciplinary board of physicians with expertise in endocrinology, endocrine oncology, endocrine surgery, radiologist, nuclear medicine, and pathology.

\section{Local Treatments}

Before starting systemic therapy with MKIs, a careful analysis of some clinical features of the patient, such as age and general health conditions, as well as some considerations about the number, the size, the site, and the rate of growth of the lesions should be performed. The reason for this reappraisal is related to the awareness of the negative impact of these drugs on the quality of life of patients. As a consequence, whenever possible a local treatment, including surgery, should be preferred, and systemic therapy should be postponed until evidence of a multimetastatic progressive disease (Fig. 1). The possibility of using the same types of local treatments should be considered also when the patient is already receiving MKI treatment with a good control of the majority of systemic disease with the exception of only one lesion. In this case, a specific locally ablative therapy directed at the growing lesion can be considered without necessarily interrupting MKI treatment temporarily. The most common types of local treatments, other than surgery, are reported below. 


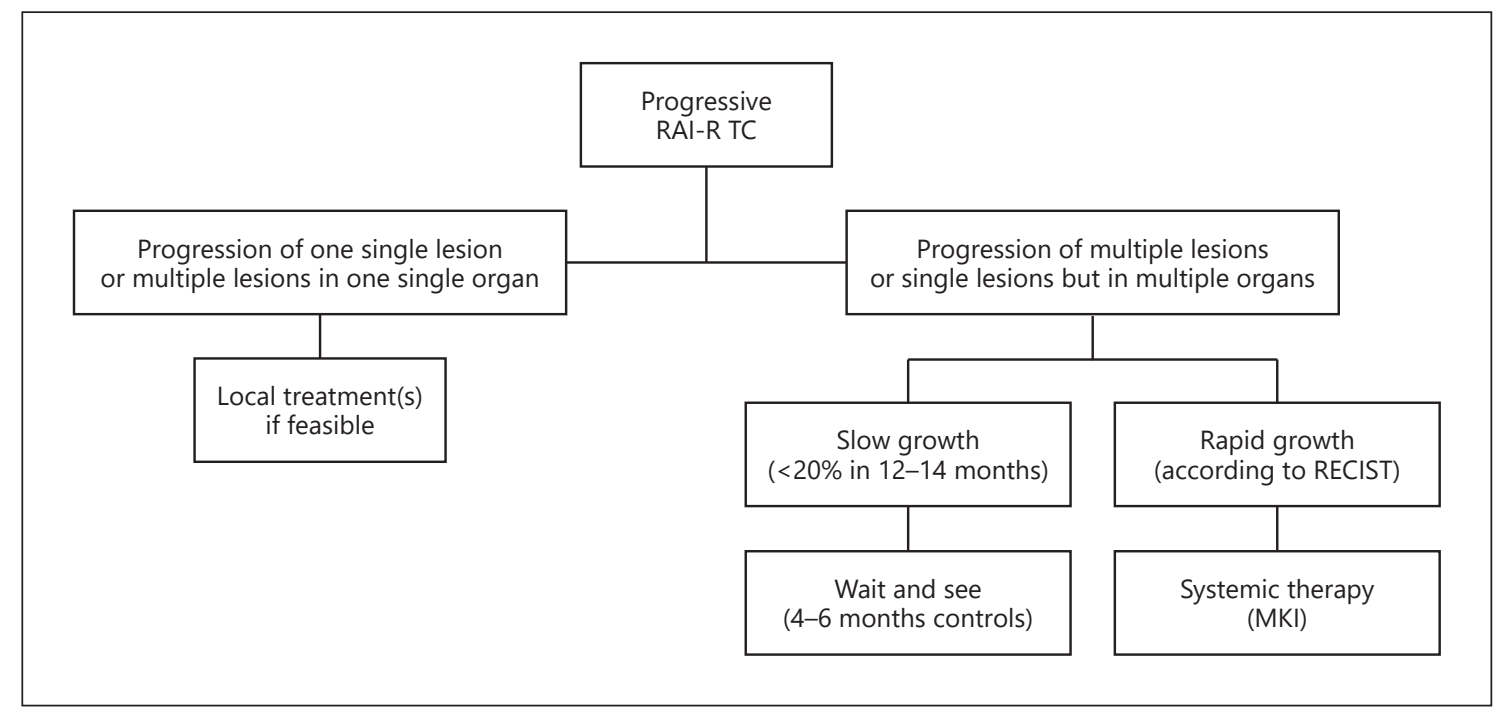

Fig. 1. Algorithm for decision making when a radioiodine refractory (RAI-R) thyroid cancer (TC) is progressing.

\section{External Beam Radiotherapy}

Although RAI-R TC are not very sensitive to radiation, external beam radiotherapy (EBRT) may be indicated in some specific cases [44]. The primary objective of EBRT in these tumours is to stop the growth or at least to reduce the rate of growth in order to control or prevent symptoms. For this reason, EBRT can be used for the treatment of inoperable primary or locally recurrent TC. Similarly, it can be considered in case of recurrent lymph node metastases after repetitive surgery. EBRT is associated with improved local disease control and a longer progressionfree survival time $[45,46]$. With the same medical objective, but also to reduce the pain, EBRT can be used in bone metastases. Brain metastases may be also treated with EBRT, with a stereotactic radiosurgery and/or wholebrain radiotherapy, and a rapid and reliable response may be obtained. The recent introduction of stereotactic EBRT has opened up the possibility of treating small metastatic lesions in any organ including lungs, for which a diffuse radiation exposure is contraindicated due to the high risk of radiation fibrosis and respiratory dysfunction [47].

\section{Treatment of Tracheal Infiltration or Compression}

When the trachea is infiltrated and tumour invasion of the tracheal lumen is found at bronchoscopy, an ablative laser treatment to minimize obstruction can be performed. If necessary, this treatment can be periodically repeated, possibly not more frequently than every 6 months. This treatment should be preferred to EBRT of the trachea in patients who are candidates for MKI treat- ment, since, in real life, patients previously submitted to external radiotherapy seem to be at risk to develop an irreparable fistula (Fig. 2). If the trachea is not infiltrated but progressively compressed by the local tumour mass, an endotracheal stent may be applied to reduce the risk of suffocation [48].

\section{Metastasectomy of Bone and Lung Metastases}

With the improvement of surgical skills and techniques, bone metastases (e.g., vertebral lesions) can be safely removed [49]. Similarly, selected lung nodules can be removed under thoracoscopy without surgical morbidity [50].

\section{Percutaneous Interventional Techniques}

They can be classified as vascular, ablative or consolidative treatments. The vascular techniques include the trans-arterial embolization and the transarterial chemoembolization (TACE) and exploit the hyper-vascular characteristic of the TC visceral or bone metastases. The ablative techniques are applied to achieve partial or complete necrosis of the neoplastic tissue by causing a dramatic increase $\left(\geq 55^{\circ} \mathrm{C}\right.$ in radiofrequency) or decrease (around $-40{ }^{\circ} \mathrm{C}$ through cryoablation) of the intra-tumoral temperature in the visceral or bone metastases. The necrotic process can be also achieved by the ultrasoundguided ethanol injection, usually in metastatic lymph nodes. Finally, the consolidative techniques are specifically applied for the treatment of the lytic bone metastases in order to prevent a pathological fracture. All the above- 
Fig. 2. Tracheal fistula (indicated by the white arrow) which occurred after 2 months of treatment with lenvatinib in a patient with advanced RAI-R TC.

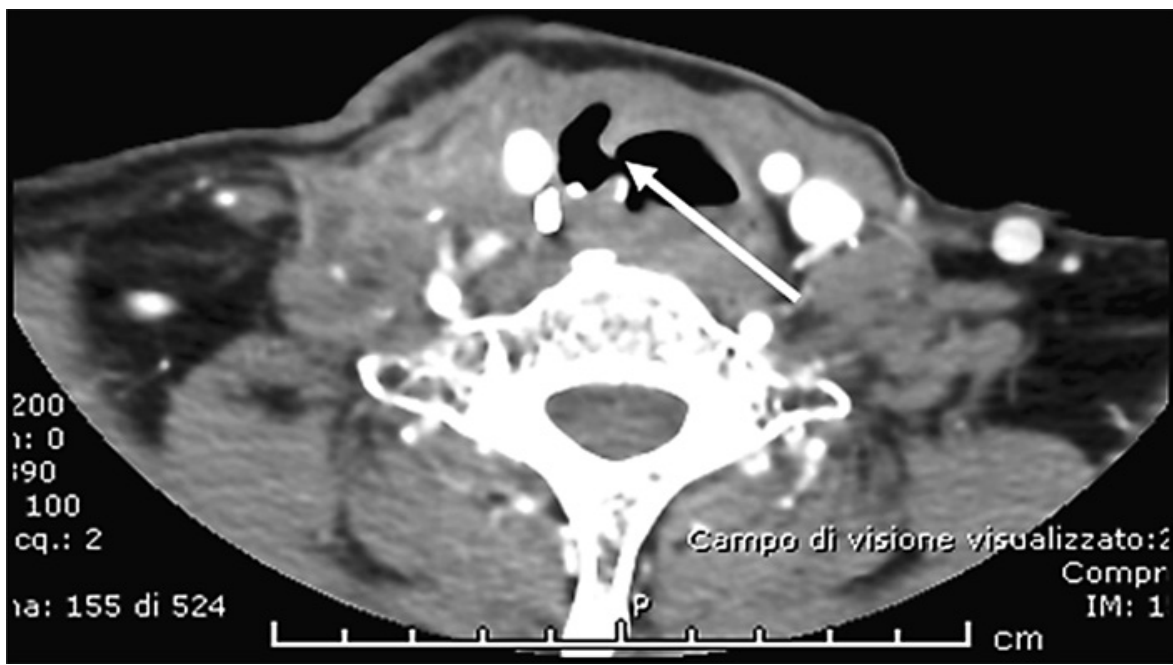

mentioned treatments can be combined and tailored according to the specific needs of the patient [51]. Some of them are more detailed below.

\section{Conventional TACE}

This technique is commonly used for palliative treatment of advanced hepatocellular carcinoma, but in some advanced cases of liver metastatic disease can also be applied in other human tumours including advanced TC. This procedure seems to have a clinical benefit when liver metastases are smaller than $3 \mathrm{~cm}$ and liver involvement is less than $30 \%$. It is yet unproven if conventional TACE used in selected patients with advanced TC is really effective at prolonging survival or at least progression-free survival [52]. Cases are relatively rare, and a prospective study is still missing [52]. Recently, the transarterial delivery of $Y 90$ radiolabelled resin microspheres, named radioembolization (or cTARE), has been successfully introduced into clinical practice for treatment of hepatocellular carcinoma and neuroendocrine tumours, but studies on liver metastasis of RAI-R TC are missing [53].

\section{Radiofrequency Thermoablation}

This procedure is based on the use of electromagnetic waves that induce movement and consequently the production of heat to destroy tumour cells. The technique was initially developed for the treatment of primary and metastatic liver malignancies [54]. This treatment can be applied to local disease, such as metastatic lymph nodes but also to bone, liver, and lung lesions, if technically possible and clinically indicated. Thus, it might be performed when the lesion to be treated is the only site of disease or the only one amongst others that is growing, and where there is no surgical option. Radiofrequency thermoablation is a safe procedure to treat the viable tumour tissue and to reduce the volume of a metastatic lesion [55], which can be afterwards submitted to surgical removal.

\section{Ultrasound-Guided Percutaneous Ethanol Ablation}

In case of neck relapse, small metastatic lymph nodes $<1.0-1.5 \mathrm{~cm}$ can be frequently found. If smaller than 1.5 $\mathrm{cm}$ in the largest diameter, they can just be periodically observed (every 6-12 months) until the evidence of a stable volume and a stable number. If growing, they can be successfully and safely treated with ultrasound-guided percutaneous ethanol ablation that can be repeated in case of recurrence [56]. This procedure is often manageable at ambulatory level, does not require hospitalization, and has a very low cost. However, it is worth to note that in advanced and progressive TC, neck lymph node metastases are often a negligible problem compared to the other types of metastases, and therefore observation may be sufficient.

\section{Cementoplasty of Bone Metastases}

It is a palliative treatment for osteolytic bone metastases, to be performed alone or in combination with other local treatments, such as radiofrequency ablation or EBRT. Its aim is to obtain the reinforcement of bone defect through percutaneous injection of polymethylmethacrylate cement. This minimally invasive technique can be performed in any metastatic site and results in significant pain relief, offering stability of the affected bones [57]. 


\section{Recommendation}

[R14] If the progression is limited to a single lesion or more than one lesion within the same organ, the possibility of performing a local treatment should be considered.

[R15] Local treatment can be also done during systemic therapy if only a single lesion starts to grow. In this case, local therapy can be considered without interrupting MKI treatment temporarily or withdrawing it just for few days.

\section{When to Start Treatment with MKIs?}

The conclusion that a patient has RAI-R disease and/ or disease progression should not automatically lead to the initiation of treatment with MKI. In answering the question whether or not initiation of MKI is beneficial, the following questions are relevant.

\section{Tumour Load}

Currently, no studies have been published on the relationship between tumour load and efficacy of MKIs. Subgroup analyses of the DECISION and SELECT studies indicated that sorafenib and lenvatinib were more effective in smaller tumours $[13,14,58]$; however, no definite cut-off level with respect to tumour size can be derived from the literature.

\section{Symptoms}

No studies are available on the relationship between clinical symptoms and efficacy of MKIs. Subgroup analyses of the DECISION and SELECT studies did not show differences in efficacy in relation to the presence of symptoms $[13,14]$. Consequently, it cannot be concluded from the literature if an MKI should be initiated before the occurrence of clinical symptoms or when clinical symptoms have occurred. However, ECOG is a major criterion with treatment being more efficient in patients with ECOG 0-1. Currently, an international prospective observational trial is being performed to address this issue [59].

\section{Location of Metastases}

No relationship has currently been identified between location of metastases and efficacy of MKIs. Subgroup analyses of the DECISION and SELECT studies did not show differences in efficacy in relation to location of metastases $[13,14]$. Brain and liver metastases might be less responsive than other sites [58].

\section{Age}

In a sub-analysis of the SELECT trial by Brose et al. [60], the safety and efficacy of treatment with lenvatinib were compared between a group of patients with a median age of 56 years, and a group of older patients with a median age of 71 years. The incidence of severe treatment-related adverse events (AEs) was significantly higher in older patients versus younger patients ( 89 vs. $67 \%$, respectively; $p<0.001$ ). Older patients were also more likely to experience AEs requiring dose modifications, and they received a lower median daily dose, though no significant difference was observed in progression-free survival between the two groups. This highlights the importance to accurately evaluate risks and benefits of MKI treatment in each patient.

\section{Genetic Profile}

Although MKIs have distinct in vitro activity in relation to molecular targets, no clear relationships have been identified between genetic profiles of TC in patients and efficacy of drugs. Subgroup analyses of the DECISION and SELECT studies did not show differences in efficacy in relation to BRAF or RAS genetic alterations $[13,14]$. The absence of these relationships can be explained by many factors, including the fact that only primary tumours have been included in genetic analyses, the existence of heterogeneity in terms of genetic profiles between metastases within one patient, many other factors that influence tumour growth, pharmacodynamics aspects, and the fact that neither sorafenib nor lenvatinib target a specific TC oncogene, but rather exert anti-angiogenic effects.

The decision on when to start treatment with MKIs should be addressed in an MDT, weighing all the benefits and disadvantages.

\section{Recommendation}

[R16] As currently no curative drug treatments for RAI-R TC are present, and MKIs are accompanied by side effects, experts agree that treatment with MKIs should only be considered in patients with progressive RAI-R disease, with considerable tumour load and when, according to a multidisciplinary group of experts, refraining from treatment with MKIs would lead to considerable harm/clinical complications within the near future. Before starting MKIs, local treatments should be considered.

[R17] The decision to start MKIs should include patient-related medical factors (age, health status, comorbidities, and contraindications) and patient preferences with respect to treatment goals and values, and acceptance of adverse effects. 


\section{Dosage}

Following the DECISION trial, approval for sorafenib was given at the dosage of $400 \mathrm{mg}(2 \times 200$ - $\mathrm{mg}$ tablets $)$ twice daily (adult patients) [13]. The drug should be taken $12 \mathrm{~h}$ apart, without food, at least $1 \mathrm{~h}$ before or $2 \mathrm{~h}$ after a meal. Following the SELECT trial, approval for lenvatinib was given at the dosage of $24 \mathrm{mg}(2 \times 10-\mathrm{mg}$ and 1 $\times 4$-mg tablets), once daily (adult patients) [14]. Capsules can be swallowed whole or, in selected cases, they can be dissolved in a small amount of water and administered with or without food intake. In case of severe renal impairment (creatinine clearance less than $30 \mathrm{~mL} / \mathrm{min}$ calculated by the Cockcroft-Gault equation using actual body weight), or in case of severe hepatic impairment (Child-Pugh C), the recommended dosage of lenvatinib for DTC patients is $14 \mathrm{mg}$ orally once.

Globally, in case of grade 3 or grade 4 AEs, it is recommended to withhold the drug until improvement to grade 0 or 1 or baseline and then to either resume at a reduced dose or to discontinue. However, this depends on the type of AE, and in some cases such as fistulas, it is recommended to withdraw permanently. For sorafenib, dosage may be reduced to $400 \mathrm{mg}$ once daily or to a single $400 \mathrm{mg}$ dose every other day. For lenvatinib, first reduction is to $20 \mathrm{mg}$, second to $14 \mathrm{mg}$, third to $10 \mathrm{mg}$. There are no data on the use of sorafenib in children, whereas lenvatinib was given in children at the dose of 14 $\mathrm{mg} / \mathrm{m}^{2}$ [61].

Data from the phase III trials showed that $64 \%$ of the patients under sorafenib and $68 \%$ of the patients under lenvatinib needed a dose reduction, $66 \%$ of the patients under sorafenib and $88 \%$ of the patients under lenvatinib required a temporary dose interruption, and $19 \%$ of the patients under sorafenib and $14 \%$ under lenvatinib needed withdrawal due to side effects. Overall, the mean dose of sorafenib received was $651 \mathrm{mg} / \mathrm{day}$, and the mean dose of lenvatinib received was $17.2 \mathrm{mg} /$ day. Although for lenvatinib a correlation was shown between the drug exposure and the percent of tumour size decrease [62], it should be noted that patients over the age of 65 years old, who represent the majority of patients with advanced TC, have more AEs than those $<65$ years old [60]. Based on these results, the question of starting lenvatinib at a lower dosage has been raised, a prospective phase II trial is ongoing comparing the dosage of $24 \mathrm{mg}$ with $18 \mathrm{mg}$, in order to evaluate if starting lenvatinib at a lower dosage decreases secondary effects without altering treatment efficacy (\#NCT02702388).

\section{Recommendation}

[R18] With the exception of patients with severe renal or liver dysfunction, treatment should be started at the approved dosage. However, active management of AEs should be organized in order to decrease the dose in case of AE. Special attention should be given to older patients because of a higher rate of AEs.

\section{How to Advise the Patient and the Family}

Decisions regarding the management of advanced RAI-R TCs are complicated and challenging for the patient and the medical team. As in all situations in medicine, a careful decision making process involving medical, emotional, social, and spiritual factors is essential to find the optimal approach for a patient. In this process, the following elements can be identified: inventory, development of management plans, information, decision making. These elements are addressed separately, but will in most cases take place in parallel.

\section{History}

Before management proposals can be formulated, information should be collected on the following items: (a) The medical state of the patient, including the disease status, physical performance, presence of comorbidities and co-medication. (b) The emotional situation of the patient. What is the degree of anxiety? Is there depression? (c) The social situation of the patient, including dependency, competences and vulnerabilities and the supportive network. (d) The personal expectations, perspectives, goals, and needs of the patient.

\section{Development of Management Plans}

Based on the above-mentioned information, integral management plans can be designed, not only focussing on medical management (including symptomatic and palliative treatment), but also addressing the social, emotional, and spiritual needs of the patient. The problem with developing medical plans is that there is limited evidence based on clinical studies that can be translated directly to the situation of the individual patient. As a consequence, developing these plans is based mostly on expert opinion and the experience of a MDT in which all healthcare specialists are represented, not restricted to medical specialists, but also general practitioners, social, psychological, and spiritual caregivers. 


\section{Information}

The patient will be informed on the items that have been inventoried as well as the treatment scenarios that have been proposed. The patient will be informed on benefits and disadvantages of the different proposals. Based on the input of the patient, scenarios can be adapted or new options developed. The amount of information that a patient is able to process depends of course on her/his emotional state but also on her/his capabilities. It is best when a patient is accompanied by relatives during these contacts.

\section{Decision Making}

All decisions in medicine, with the exception probably of acute, life-threatening conditions, are shared decisions, meaning that opinions of patients and healthcare professionals are brought together. In the end, the patient is the person who has to make a decision, but the role of the patient in the process of decision making may vary greatly. Most patients however, do not want to receive only data and facts, but expect a clear preference from their doctors. Patients will need time to weigh all the information and management options and often want to consult relatives or experts.

\section{Recommendation}

[R19] Gather extensive information on the patients' psychophysical status before formulating management proposals.

[R20] Develop management plans mostly based on expert opinion of an MDT.

[R21] Inform patient and relatives on benefits and disadvantages of the different available management plans and together decide on appropriate management for the individual patient.

\section{Duration of Treatment and Follow-Up}

\section{Duration of Treatment}

Tyrosine kinase inhibitors are cytostatic, and not cytotoxic drugs; thus, they exert a break to the cell growth that can restart immediately when the break is removed $[63,64]$. This is the rationale why an MKI therapy should be continued until the evidence of no further clinical benefit. The concept of clinical benefit is not well defined, but we can recapitulate it according to a series of clinical factors such as: (a) the evidence of partial remission or stabilization of the tumour burden, (b) the improvement of symptoms, (c) the slow progression of the disease or the progression of only one lesion while the others are stable. Moreover, there is evidence, at least for sorafenib, that patients who entered into progression and continued to be treated with the drug showed a slower progression than those who stopped the drug [25]. On the basis of this evidence, the best option for the patient is to continue the drug at least until the evidence that a second line of MKI or a clinical trial with new experimental drugs is available and, at this point, it could be useful to switch the therapy $[64,65]$. Sometimes, MKI therapy leads to severe side effects and if the degree of severity, according to the Common Terminology Criteria for Adverse Events (CTCAE) scale, is $\geq 3$, the drug must be stopped until recovery from the side effects, but then it should be restarted at a lower daily dose. However, patients must be clearly instructed to report side effects even when they are still low grade in order to avoid MKI interruption. Overall dose reduction, which can be effective when a side effect is still low grade, should be preferred. The final objective is to find a daily dose that can provide a good balance between the quality of life of the patient and the positive effect of the drug.

\section{Follow-Up}

The follow-up of patients treated with MKI is crucial to the success of this therapy. In particular, it is fundamental that the patients have a facilitated access to reach either specialist doctors or nurses who must have been trained to follow them with their specific clinical and psychological problems. Patients treated with MKIs must be strictly followed especially during the first 6 months of therapy. This need is related to the multiple side effects that the patients can experience (see also the section Management of Side Effects). Blood pressure should be well controlled before starting the MKI, especially when starting lenvatinib [14]: if pathologically altered or even if borderline, a specific therapy must be started and then tightly controlled. Patients must be educated in doing this by $\mathrm{him} /$ herself, and a daily record should be kept in form of a diary to show to doctors or nurses at each control. A similar approach should be taken for prevention of palmar-plantar erythrodysaesthesia ("hand and foot syndrome") when sorafenib is started [13]. Patients should be educated in preventive care of hands and feet with adequate procedures and periodic controls by physicians or specialist nurses. Patients must also be taught to report any side effect as soon as possible, and for this reason they must have a direct access for communication with the doctors or specialist nurses.

Other than this advice, patients should meet their specialist's team every 2 weeks for the first 2 months. During 
Table 1. Parameters to be evaluated at baseline and at each control visit

Parameters to be evaluated

\begin{tabular}{|c|c|}
\hline $\begin{array}{l}\text { Clinical } \\
\text { data }\end{array}$ & $\begin{array}{l}\text { Weight, appetite, fatigue, diarrhoea, skin } \\
\text { manifestations, patient's diary on side effects and/ } \\
\text { or symptoms }\end{array}$ \\
\hline Blood tests & $\begin{array}{l}\text { TSH, fT4 (thyroglobulin, Tg antibodies at } \\
\text { periodical intervals) } \\
\text { Electrolytes (Ca, Na, K, Mg) } \\
\text { Full blood count } \\
\text { ALT, ALP, GGT } \\
\text { Glucose, total HDL, LDL cholesterol, triglycerides }\end{array}$ \\
\hline $\begin{array}{l}\text { Cardiac } \\
\text { parameters }\end{array}$ & $\begin{array}{l}\text { ECG (QTc interval) } \\
\text { Blood pressure }\end{array}$ \\
\hline Imaging & $\begin{array}{l}\text { Whole-body imaging (CT scan with contrast } \\
\text { medium and/or MRI and/or 18-FDG-PET/CT } \\
\text { scan) (first visit, then periodical evaluation } \\
\text { metastatic sites) } \\
\text { Bone scintigraphy (optional) }\end{array}$ \\
\hline
\end{tabular}

these visits, body weight must be controlled tightly to verify weight loss as a frequent consequence of MKI (i.e., due to anorexia, dysgeusia, mucositis, etc.) (see also the section Management of Side Effects). Furthermore, controls must include blood pressure measurement and clinical chemistry, in particular TSH and electrolytes, which can be severely altered by MKIs (Table 1). An ECG must be also performed to verify the QTc, especially when lenvatinib is administered.

Two to 3 months after the initiation of the MKI therapy, an appropriate imaging (usually by CT and/or MRI) should be performed to verify the response to the drug. An 18-FDG PET/CT could also be useful to verify the reduction of the metabolic activity of the metastatic lesions, though whether this has a prognostic or management impact still needs to be demonstrated. During these visits, a measurement of serum $\mathrm{Tg}$ concentrations and $\mathrm{Tg}$ antibodies should be done and evaluated together with the results of the imaging.

After the first 2 months, the follow-up visits may take place at monthly intervals until the 6th month and then every 3 months until the completion of the 1st year of MKI treatment. Afterwards, if patients are stable, followup intervals can be extended to 4 months with periodical imaging. The levels and the trend of the serum Tg can be very useful to plan the timing of the controls and in particular of the imaging.

2019 ETA Guidelines for the Treatment of Advanced RAI-R TC

\section{Recommendation}

[R22] MKI treatment should be continued as long as there is evidence of clinical benefit.

[R23] MKI should be stopped only when another therapeutic option is available or the side effects are intolerable or treatment is no longer providing clinical benefit or at patients' request.

[R24] In case of side effects, MKI daily dose should be reduced to maintain a balance between a good quality of life and control of disease.

[R25] Patients must be strictly followed during the first 2 months of therapy with blood tests, ECG, and clinical monitoring.

[R26] After 2-3 months of therapy, a first imaging evaluation should be performed to verify the effectiveness of MKI.

[R27] Follow-up visits should be performed every 2 weeks during the first 2 months of MKI therapy, and then every month until the first 6 months, every 3 months within the first year, and after that every 4 months.

\section{Management of Side Effects}

Virtually all MKI-treated patients experience at least one or more AE. The severity of AEs is commonly scored according to Common Terminology Criteria for Adverse Events (CTCAE version 4.03 can be downloaded without charge at https://evs.nci.nih.gov/ftp1/CTCAE/CTCA E_4.03/C TCA E_4.03_2010-06-14_ QuickReference_8.5x11.pdf. Although most AEs are manageable, some of them are potentially serious and rare, or unreported adverse effects, possibly life-threatening, could occur. Interestingly, AEs are less frequent in patients $<65$ years of age [60]. The most frequent AEs for the 2 MKIs most widely used in Europe, sorafenib and lenvatinib, are reported in Table 2.

In the majority of cases, the frequency of the AEs reported in the phase II $[66,67]$ and in the phase III registration (DECISION and SELECT) studies $[13,14]$ corresponds to the frequency found in the subsequent reallife studies. Among the most frequent AEs ( $\geq 50 \%$ of patients), are hypertension, particularly in patients taking lenvatinib, diarrhoea, weight loss, and fatigue. Dermatological AEs, such as palmar-plantar erythrodysaesthesia syndrome, skin rash, and alopecia are more frequent during sorafenib therapy, while mucositis/ stomatitis seem to occur more often during lenvatinib treatment. AEs occur at a median time of 3-12 weeks after the start of the MKIs and, generally, the frequencies of AEs are higher in the earlier cycles of treatment and diminish over the course of treatment [29], indicating that they may be self-limiting in some cases. The response to toxicities should include therapies to rapidly 
Table 2. Frequency of the most frequent adverse events in patients treated with sorafenib and lenvatinib, according to phase II, phase III registration, and real life studies [13, 15-19, 29, 65, 66]

\begin{tabular}{|c|c|c|c|}
\hline Side effect & Grade & Frequency on sorafenib, $\%^{\mathrm{a}}$ & Frequency on lenvatinib, $\%^{\mathrm{a}}$ \\
\hline \multirow[t]{2}{*}{ Hypertension } & Any grade & $41 / 31(19-42)$ & $68 / 75(67-80)$ \\
\hline & Grade 3-4 & $10 / 20(2-38)$ & $42 / 32(10-49)$ \\
\hline \multirow[t]{2}{*}{ Diarrhoea } & Any grade & $69 / 54(47-63)$ & $59 / 58(45-67)$ \\
\hline & Grade 3-4 & $6 / 7(1-13)$ & $8 / 25(1-42)$ \\
\hline \multirow[t]{2}{*}{ Weight loss } & Any grade & $47 / 30(1-58)$ & $46 / 68(46-92)$ \\
\hline & Grade 3-4 & $6 / 9(0-17)$ & $10 / 11(5-17)$ \\
\hline \multirow[t]{2}{*}{ Fatigue } & Any grade & $50 / 50(30-69)$ & $59 / 55(44-61)$ \\
\hline & Grade 3-4 & $6 / 7$ & $9 / 8(5-10)$ \\
\hline \multirow[t]{2}{*}{ Anorexia/nausea } & Any grade & $32 / 13(7-21)$ & $41 / 50(18-75)$ \\
\hline & Grade 3-4 & $2 / 1$ & $2 / 4(1-8)$ \\
\hline \multirow[t]{2}{*}{ Mucositis/stomatitis } & Any grade & $23 / 38(27-48)$ & $36 / 41(24-58)$ \\
\hline & Grade 3-4 & $1 / 20$ & $4 / 5(2-8)$ \\
\hline \multirow{2}{*}{$\begin{array}{l}\text { Palmar-plantar } \\
\text { erythrodysaesthesia }\end{array}$} & Any grade & $76 / 72(69-76)$ & $32 / 29(26-33)$ \\
\hline & Grade 3-4 & $20 / 26(20-32)$ & $3 / 2(1-3)$ \\
\hline \multirow[t]{2}{*}{ Proteinuria } & Any grade & - & $31 / 44(32-64)$ \\
\hline & Grade 3-4 & - & $10 / 12(10-13)$ \\
\hline \multirow[t]{2}{*}{ Skin rash } & Any grade & $50 / 38(25-55)$ & $16 / 16$ \\
\hline & Grade 3-4 & $5 / 16(3-29)$ & $0.4 / 1$ \\
\hline \multirow[t]{2}{*}{ Alopecia } & Any grade & $67 / 38(31-52)$ & - \\
\hline & Grade 3-4 & - & - \\
\hline \multicolumn{2}{|l|}{ Increased TSH } & $33 / 26$ & $-/ 44(21-67)$ \\
\hline
\end{tabular}

and effectively reduce the AEs. Nevertheless, there are some AEs such as weight loss and anorexia that are very difficult to manage and require the reduction of the daily dose, if not the complete interruption of the drug, though every effort should be made to optimize tolerance and maintain the highest dosage level that is reasonably tolerable and effective in each patient.

\section{Hypertension}

It is a frequent $\mathrm{AE}$ of MKI treatment and often is grade 2 or 3. Interestingly, recent data in patients with RAI-R TC treated with lenvatinib showed that hypertension significantly correlated with improved outcomes, indicating that it may be predictive of drug efficacy [68]. Blood pressure and left ventricular ejection fraction should be measured and optimized before starting treatment. The choice of the antihypertensive drug should be individualized, using angiotensin-converting enzyme inhibitors and/or calcium channel blockers, alone or in association, as the first-line drugs. If this is not effective, a diuretic should be added, electrolytes monitored, and dehydration avoided. Patients should check their blood pressure at home, with a daily or biweekly frequency, in order to allow a closer monitoring and titration of antihypertensive therapy, with a target blood pressure $\leq 140 / 90 \mathrm{~mm}$ $\mathrm{Hg}$ (lower in case of overt proteinuria).

\section{Diarrhoea}

It is an extremely frequent $\mathrm{AE}$, involving around $80 \%$ of patients taking MKIs, and is likely one of the most disabling when the degree of severity is high [13]. It occurs mostly as grade 1 or grade 2 , has a slow onset, and is typically intermittent ( 2 or 3 episodes per week) $[26,29]$. When the degree of severity is $<3$, it can be managed with dietary adjustments, with fluid and electrolyte replacement, usually accompanied by pharmacological interventions (such as loperamide, amisulpride, codeine). Some patients report benefits upon intermittent or prophylac- 

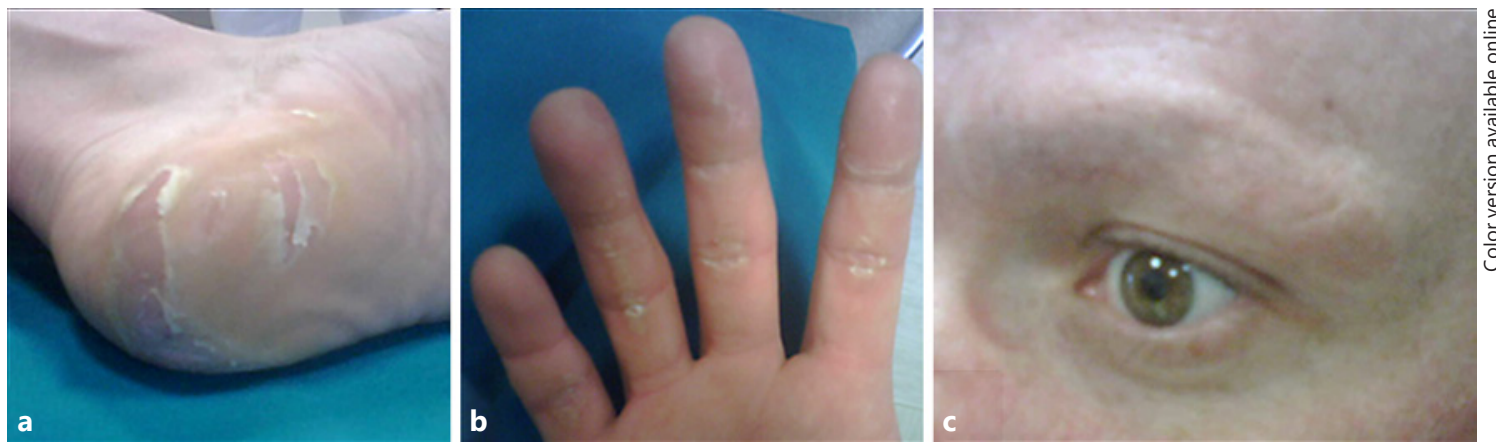

Fig. 3. Palmar-plantar erythrodysaesthesia syndrome ("hand-foot-syndrome") (a and b) and alopecia of the eyebrows $(\mathbf{c})$ in a patient treated with sorafenib.

tic consumption of natural remedies such as aloe or probiotics. In case of grade 3 or 4 , a dose reduction or interruption until diarrhoea returns to grade 1 is needed.

\section{Weight Loss}

It is one of the most important AEs on MKIs, particularly frequent in the real-life experiences with lenvatinib (Table 2). Anorexia in general or limited to some foods is frequent, and together with diarrhoea is one of the main causes of weight loss. Further AEs can contribute to weight loss, such as dysgeusia, oral mucositis, dyspepsia, and nausea which can improve by treatment with metoclopramide or serotonin receptor antagonists. MKIs seem to mostly affect muscular mass, but the fat mass is involved too [69-71]. Anorexia and weight loss should be carefully managed by nutritional assessment at baseline and then periodically, always with the support of an expert nutritionist. Oral protein-rich, hypercaloric supplements (200-250 kcal/100 ml) should be given at the first demonstration of weight loss, eventually associated with antioxidants. Enteral nutrition (1,000-1,200 kcal/L) should be given in more severe cases.

\section{Fatigue}

It is one of the most common and probably one of the most disabling AE observed during MKI treatment. In the registration studies $[13,14]$ as well as in real-life studies [15-19], fatigue was recorded in around 50-70\% of patients, and it was a common reason for drug discontinuation or dose reduction. Several recommendations are given to patients, such as exercising (to maintain muscle mass), taking caffeine, MKI intake in the evening, monitoring electrolytes, haemoglobin, and $\mathrm{TSH}$, maintaining hydration and adequate food intake, but in most cases these recommendations are not effec- tive. It has been recently reported that primary adrenal insufficiency (PAI) occurs frequently in patients treated with lenvatinib, and that replacement treatment with cortisone acetate consequently leads to relief of fatigue [72].

\section{Dermatological AEs}

Cutaneous alterations, such as the palmar-plantar erythrodysaesthesia syndrome ("hand-foot syndrome"), are rare during lenvatinib treatment, but extremely frequent under sorafenib, being the most common reason for dose reduction, interruption, and withdrawal [13] (Fig. 3).

The intensity is variable and increases in the first months of therapy with a progressive decrease thereafter. The skin of hands and feet should be in optimal conditions before starting MKI treatment by using urea-based creams and ensuring removal of hyperkeratotic lesions from hands and feet prior to MKI therapy. Management includes keeping hands and feet well hydrated, wearing comfortable shoes, avoiding hot water and trauma, removing calluses, and using pain medication as needed. In more severe cases, ibuprofen (600 mg three times a day) can be prescribed, though dose reduction or short interruptions of MKIs can be required. Skin rash/sun burn after outdoor activities are also underestimated AEs and can be prevented by avoiding direct sunlight exposure, wearing protective clothing, including a hat and using sunblock ( $\mathrm{UF} \geq 50$ ).

\section{Mucositis/Stomatitis}

Oral AEs usually occur within 2-4 weeks of initiating therapy. Oral hygiene and frequent mouthwash with water and baking soda, high-molecular-weight hyaluronic acid, or aloe vera extracts are recommended. Topical an- 

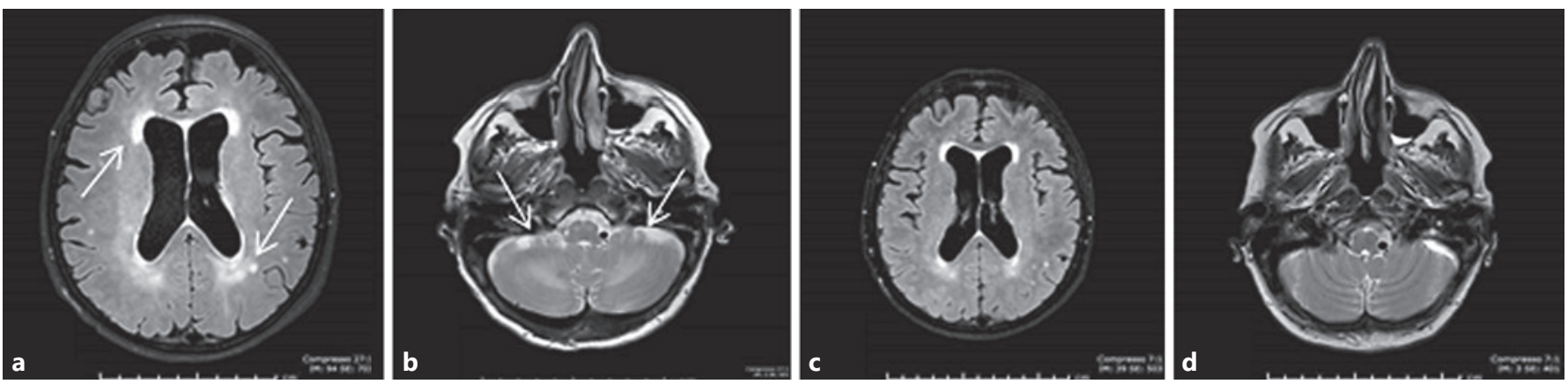

Fig. 4. The rare adverse event called reversible posterior leukoencephalopathy syndrome. a, b Brain MRI showed a posterior periventricular white matter hyperintensity and a signal alteration of the white matter interior of the cerebellum, confirming the diagnosis of toxic encephalopathy. c, d Brain MRI performed after 2 weeks of lenvatinib withdrawal documented an almost complete resolution of the radiological alterations, allowing to re-introduce the MKI.

algesics alone or in combination (lidocaine $2 \%$, diphenhydramine, bismuth subsalicylate, aluminum or magnesium hydroxide) can be used in more severe cases.

\section{Proteinuria}

Baseline and periodic assessment of urinary protein excretion is mandatory particularly in lenvatinib treatment. Angiotensin-converting enzyme inhibitors or angiotensin receptor antagonist are generally effective. In the rare occurrence of nephrotic syndrome, MKI treatment must be temporarily discontinued [73].

\section{Increase in TSH Levels}

Thyroid function should be monitored every 1-2 months since thyroxine dosage needs to be increased in many TC patients on MKIs [74, 75].

\section{Delay in Wound Healing}

Inhibition of the VEGFR signalling pathway impairs angiogenesis and can delay the wound healing process. It is thus recommended to withdraw MKI treatment 3-10 days before and after surgery or tooth extraction, depending on the extent of the procedure.

\section{Rare AEs}

Some rare AEs are the consequence of MKI-induced hypertension, such as congestive heart failure [76], haemorrhage, and cardiac infarction [77]. Arterial thrombotic events, in particular acute coronary syndrome, require immediate cardiological treatment. The occurrence of arterial thrombotic events may require permanent drug withdrawal. Venous thrombotic events should be managed by low-molecular-weight heparin. Alterations in blood cell count can occur during MKI treatment, and in most cases are grade 1 and 2 AEs $[67,78]$. Arthralgias and myalgias occur in around $10 \%$ of patients and can be managed by analgesia and if required corticosteroid therapy. Hoarseness has been reported in around $15 \%$ of lenvatinib-treated patients.

A rare but severe AE is the reversible posterior leukoencephalopathy syndrome. At onset, neurological symptoms can be observed, and alterations of the white matter, consistent with toxic encephalopathy, can be documented on cranial MRI. MKI treatment must be stopped and a "restitution ad integrum" is usually observed within 2-3 weeks (Fig. 4). A range of potentially serious gastrointestinal (GI) tract AEs, such as haemorrhage, intestinal perforation and GI and non-GI (i.e., tracheal or oesophageal) fistulas can be observed [79]. The risk of intestinal perforation with MKIs is probably higher in patients with a history of bowel conditions, such as colitis or diverticulitis, and tracheal or oesophageal fistulas preferentially occur at site of previous surgery or irradiation. Treatment must be discontinued if a perforation or a fistula occurs. Lenvatinib-induced renal failure has recently been reported, due to glomerular damage with proteinuria or tubulointerstitial injury without proteinuria [80].

The broad range and potential severity of MKI-related toxicities underscore the importance of proactive monitoring for, early recognition of, and expeditious response to emerging toxicities. Education should be provided to each patient before starting an MKI treatment. Some of the expected AEs can be prevented with an appropriate patient history and physical examination and with the precise information of the patient and of her/his family 
members, e.g. as documented in the patient's diary (see also the section Duration of Treatment and Follow-Up). Appropriate actions should be taken as soon as the symptoms appear, in order to promptly and effectively manage the $\mathrm{AE}$, thus avoiding drug discontinuation.

\section{Recommendation}

[R28] An accurate multidisciplinary evaluation of the clinical features of the patient should be performed before starting MKIs.

[R29] Patient and carer education regarding potential toxicities and their management should be provided prior to initiation of treatment.

[R30] Patients must be instructed to report AEs, even when they are still low grade.

[R31] Patients must be treated by a TC specialist within an MDT and timely and effective supportive care and MKI dose adjustment should be given as needed.

[R32] Clinical and biochemical controls (including clinical examination, evaluation of AEs, blood count, urine analysis, renal and hepatic function, TSH, calcium) must be performed every 2 weeks during the first 2 months of treatment.

[R33] In order to limit the number of visits and to increase the compliance of patients, it is suggested to establish a dedicated contact number.

[R34] Every effort and intervention should be made in order to limit dose reduction and to avoid drug discontinuation.

\section{Palliative Treatments}

The vast majority of TC cases are diagnosed at an early stage when it is curable. However, RAI-R DTC has a poor prognosis with a median survival of less than 5 years and with disease progression is frequently associated with a reduction in quality of life due to debilitating symptoms.

Advanced TC presents specific management issues. The anatomical site of locally advanced disease in the neck may cause airway compromise, dysphagia, and dysphonia and is often visible providing a constant reminder to the patient of their disease. Distant metastatic disease causes symptoms according to site such as pain, skeletal fracture, breathlessness, or organ obstruction. Some patients will have been living with their disease in an indolent phase for many years, and the psychological impact of accepting the change in the nature of their disease to an aggressive, symptomatic phase can be difficult to deal with.

Specialists in palliative care medicine have expertise in managing multiple complex symptoms, psychosocial support, and assistance with decision making [81]. The involvement of the palliative care team in a patient's man-

2019 ETA Guidelines for the Treatment of Advanced RAI-R TC agement at an earlier time than the end of life phase is extremely beneficial in terms of both quality and quantity of life for the patient [81] and their carers. A relationship can be developed between clinician and patient with an understanding of patients' wishes and concerns. This should lead to a more controlled and hopefully more comfortable life with advanced disease.

The typical long natural history of the disease means that many patients will have had multiple treatments over many years, and hence the management of advanced TC requires a case-by-case approach with an individualized management plan. An assessment of the patient should include a full history of the current symptoms, impact on quality of life and any concerns or worries the patient may have. Specific symptoms may be addressed with locally ablative therapies that will not alter the overall natural history of the disease but may provide significant symptom relief. Modalities such as surgery, radiotherapy, vertebroplasty, and interventional radiology including radiofrequency ablation and embolization may all be considered either alongside systemic therapies or alone $[44,47,52,54]$ (see also the section Local Treatments). Intervention for symptomatic, or imminently problematic, metastases in advanced TC should be considered because prognosis may still be measured in years, and therefore symptom control is critical to ensuring an optimal quality of life. Moreover, if individual sites of problematic disease can be controlled locally, the initiation of systemic therapies and their associated toxicities may be delayed. Systemic therapies for advanced disease are discussed in the sections When to Start Treatment with MKIS? to Management of Side Effects. In addition to targeted therapies to modulate the disease process, a systemic approach to bone metastases should be considered. Bone metastases can be detected in around $4 \%$ of patients and a skeletal-related event (SRE), which is an independent poor prognostic factor, in around 5.5\% [82]. Medical management with bisphosphonates, specifically zoledronate, is recommended based on data extrapolated from other solid tumours [83, 84], and a beneficial effect has been documented also in TC patients [85]. There is some evidence that denosumab, a human, monoclonal, IgG2 antibody that binds to RANKL and inhibits osteoclasts, bone destruction, and tumour growth, is more effective than zoledronate in preventing SREs in solid tumours $[86,87]$. It is worth noting that denosumab can cause hypocalcaemia and osteonecrosis of the jaw, with a higher risk if the patient is on MKIs, but has less impact on renal function and fewer acute phase reactions. 


\section{Recommendation}

[R35] Early involvement of the palliative care team is encouraged to optimize symptom control and psychosocial support in patients with advanced TC.

[R36] Consideration should be given to locally ablative therapies to palliate specific symptoms.

[R37] Consideration should be given to the systemic management of bone metastases to minimize SREs.

\section{Ethical Considerations}

ATA Clinical and Professional Ethics Guidelines for The Practice of Thyroidology, published in 2013, summarized the ethics principles that physicians should follow in their clinical practice [88]. Daily ethical duties to patients should be based on equality, competence, cultural values, physician-patient communication and should consider conflicts of interest, sexual relationships, privacy and confidentiality, treatment of family members (physicians should not treat their families), and maintaining ethical integrity in unethical environments [88].

Informed consent, involving full information on disease status, planned treatment, its consequences and possible side effects, plays a crucial role in a treatment decision-making process. One should always respect the preference of autonomous patients, their acceptance or refusal of recommended therapies. On the other hand, any pressure from family members or from a patient, to treat regardless the lack of its efficacy or potential risk, should have no impact on the decision-making process.

There are some important ethical issues that the physicians managing advanced TCs have to face. The first one is how to inform the patients about a potentially lethal disease. According to the ATA Guidelines, the following issues should be discussed: prognosis, both with and without treatment, therapeutic options, so-called "code status" (patient decision regarding cardiopulmonary resuscitation, nutrition, hydration, and intubation), palliative care, hospice care, physical/medical order for life-sustaining treatment, and other patient's "advance directives" [88]. The decision on the appropriateness of a palliative treatment, or of a cardiopulmonary resuscitation, should be physicians' responsibility, though patient's opinion should be considered. Our ethical responsibility is to provide patients with objective, evidence-based information related to their individual disease status rather than assuming what is the best for an individual patient by selectively withholding known information [89]. However, one has to be aware that precise data on a poor prognosis and lim- ited treatment options might be particularly stressful for the patients and extremely difficult to accept and simultaneously not to lose hope. Therefore, the support of a psychologist and/or of a specialized nurse should be considered and offered to the patients. In this context, it is also worth mentioning that patients with advanced disease should be treated at or in collaboration with specialized centres with all needed disciplines available.

Other important clinical questions to consider involve: "to treat or not to treat," "which treatment to choose: conventional or experimental" or "when to start or stop the treatment."

Answering the questions "to treat or not to treat" and "which treatment to choose: conventional or experimental" one should consider evidence-based data on treatment efficacy, its impact on progression-free and overall survival, and on the quality of life, life expectancy, comorbidities, and risk/benefits ratio. The question "when to start the treatment" may also infer ethical dilemma. It should be emphasized that evidence-based medicine supports treatment initiation in patients with bulky, immediately life-threatening or progressive disease. Another question "when to stop the treatment" seems to be even more important. Is disease progression a sufficient condition to stop the treatment? It is believed that the withdrawal of MKI may speed up disease progression; hence, it could be better to continue treatment, if there are no other therapeutic options, than to withdraw it. However, since MKI treatment is not inexpensive, this decision can pose an ethical dilemma.

\section{Recommendation}

[R38] The management of patients with advanced RAI-R TC should respect general ethical principles, such as: equality, competence, cultural values, physician-patient communication, privacy and confidentiality, and conflicts of interest. Physicians are obliged to respect autonomous patients' preference.

[R39] Once approved therapies have failed or if there are reasons for rejecting them, patients with advanced TC should be treated preferably within randomized clinical trials to provide evidence-based data on adequate therapeutic approach.

\section{Conclusions and Perspectives}

Advanced RAI-R TC represents a challenging condition. In the last decade, novel local and systemic treatments have been made available to specialists. However, the real-life practice has taught us that these complex TC 
patients must be managed by a multidisciplinary board of physicians, and always by specialized nurses. The board should initially include specialists in endocrinology, endocrine oncology, endocrine surgery, pathology, radiology, nuclear medicine, and interventional radiology. After the start of the MKI treatment, the board should also include specialists in psychology, nutritional medicine, dermatology, and cardiology.

Several novel and promising perspectives are available for the treatment of advanced TC. Ongoing studies are evaluating the efficacy and safety of new MKIs, redifferentiation therapies, and of immunotherapeutic compounds to be used alone or in combination, as first or second and third-line treatments.

\section{Disclosure Statement}

L.F. consults for Eisai Europe Limited, Sanofi-Genzyme. R.E. consults for Eisai Europe Limited, Sanofi-Genzyme, Exilixis, and LOXO. D.F. consults for Eisai Europe Limited, Sanofi-Genzyme, Ipsen, and Novartis. B.J. is a member of AstraZeneca and Sobi Advisory Boards; has received honoraria from Amgen, Bayer Health Care, Eisai, Exelixis, Ipsen, Novartis, Oxigene, Pfizer, and SanofiGenzyme. S.L. is a member of Sanofi Genzyme, EISAI, Loxo, and Bayer Advisory Boards; has received research grants from Sanofi Genzyme, Novartis, and Bayer. K.N. consults for Eisai Europe Limited, Sanofi-Genzyme. J.S. has no conflicts to disclose.

\section{References}

1 Lloyd RV, Osamura RY, Klöppel G, Rosai J, editors. WHO Classification of Tumours of Endocrine Organs. 4th ed. IARC WHO Classification of Tumours. Lyon: IARC; 2017.

2 Volante M, Collini P, Nikiforov YE, Sakamoto A, Kakudo K, Katoh R, et al. Poorly differentiated thyroid carcinoma: the Turin proposal for the use of uniform diagnostic criteria and an algorithmic diagnostic approach. Am J Surg Pathol. 2007 Aug;31(8):1256-64.

3 Ibrahimpasic T, Ghossein R, Carlson DL, Nixon I, Palmer FL, Shaha AR, et al. Outcomes in patients with poorly differentiated thyroid carcinoma. J Clin Endocrinol Metab. 2014 Apr;99(4):1245-52.

4 Baloch ZW, LiVolsi VA. Special types of thyroid carcinoma. Histopathology. 2018 Jan; 72(1):40-52.

5 Volante M, Bussolati G, Papotti M. The story of poorly differentiated thyroid carcinoma: from Langhans' description to the Turin proposal via Juan Rosai. Semin Diagn Pathol. 2016 Sep;33(5):277-83.

6 Dettmer M, Schmitt A, Steinert H, Moch H, Komminoth P, Perren A. Poorly differentiated oncocytic thyroid carcinoma-diagnostic implications and outcome. Histopathology. 2012 Jun;60(7):1045-51.

7 Rivera M, Ghossein RA, Schoder H, Gomez D, Larson SM, Tuttle RM. Histopathologic characterization of radioactive iodine-refractory fluorodeoxyglucose-positron emission tomography-positive thyroid carcinoma. Cancer. 2008 Jul;113(1):48-56.

8 Haugen BR. Management of the patient with progressive radioiodine non-responsive disease. Semin Surg Oncol. 1999 Jan-Feb;16(1): 34-41.

9 Agrawal N, Akbani R, Aksoy BA, Ally A, Arachchi H, Asa SL, et al.; Cancer Genome Atlas Research Network. Integrated genomic characterization of papillary thyroid carcinoma. Cell. 2014 Oct;159(3):676-90.
10 Landa I, Ibrahimpasic T, Boucai L, Sinha R, Knauf JA, Shah RH, et al. Genomic and transcriptomic hallmarks of poorly differentiated and anaplastic thyroid cancers. J Clin Invest. 2016 Mar;126(3):1052-66.

$11 \mathrm{Xu} \mathrm{B}$, Ghossein R. Genomic Landscape of poorly Differentiated and Anaplastic Thyroid Carcinoma. Endocr Pathol. 2016 Sep;27(3): 205-12.

12 Durante C, Haddy N, Baudin E, Leboulleux S, Hartl D, Travagli JP, et al. Long-term outcome of 444 patients with distant metastases from papillary and follicular thyroid carcinoma: benefits and limits of radioiodine therapy. J Clin Endocrinol Metab. 2006 Aug;91(8):2892-9.

13 Brose MS, Nutting CM, Jarzab B, Elisei R, Siena S, Bastholt L, et al.; DECISION investigators. Sorafenib in radioactive iodine-refractory, locally advanced or metastatic differentiated thyroid cancer: a randomised, double-blind, phase 3 trial. Lancet. 2014 Jul; 384(9940):319-28.

14 Schlumberger M, Tahara M, Wirth LJ, Robinson B, Brose MS, Elisei R, et al. Lenvatinib versus placebo in radioiodine-refractory thyroid cancer. N Engl J Med. 2015 Feb;372(7):62130.

15 Berdelou A, Borget I, Godbert Y, Nguyen T, Garcia ME, Chougnet CN, et al. Lenvatinib for the Treatment of Radioiodine-Refractory Thyroid Cancer in Real-Life Practice. Thyroid. 2017 Nov; [Epub ahead of print].

16 Balmelli C, Railic N, Siano M, Feuerlein K, Cathomas R, Cristina V, et al. Lenvatinib in Advanced Radioiodine-Refractory Thyroid Cancer - A Retrospective Analysis of the Swiss Lenvatinib Named Patient Program. J Cancer. 2018 Jan; 9(2):250-5.

17 Nervo A, Gallo M, Samà MT, Felicetti F, Alfano M, Migliore E, et al. Lenvatinib in Advanced Radioiodine-refractory Thyroid Cancer: A Snapshot of Real-life Clinical Practice. Anticancer Res. 2018 Mar;38(3):1643-9.
18 Kim M, Kim TH, Shin DY, Lim DJ, Kim EY, Kim WB, et al.; Korean Thyroid Cancer Study Group (KTCSG). Tertiary care experience of sorafenib in the treatment of progressive radioiodine-refractory differentiated thyroid carcinoma: A Korean multicenter study. Thyroid. 2018 Mar;28(3):340-8.

19 Molina-Vega M, García-Alemán J, SebastiánOchoa A, Mancha-Doblas I, Trigo-Pérez JM, Tinahones-Madueño F. Tyrosine kinase inhibitors in iodine-refractory differentiated thyroid cancer: experience in clinical practice. Endocrine. 2018 Feb;59(2):395-401.

20 Haugen BR, Alexander EK, Bible KC, Doherty GM, Mandel SJ, Nikiforov YE, et al. 2015 American Thyroid Association Management Guidelines for Adult Patients with Thyroid Nodules and Differentiated Thyroid Cancer: The American Thyroid Association Guidelines Task Force on Thyroid Nodules and Differentiated Thyroid Cancer. Thyroid. 2016 Jan;26(1):1-133.

21 Schlumberger M, Brose M, Elisei R, Leboulleux S, Luster M, Pitoia F, et al. Definition and management of radioactive iodine-refractory differentiated thyroid cancer. Lancet Diabetes Endocrinol. 2014 May;2(5):356-8.

22 Luster M, Clarke SE, Dietlein M, Lassmann M, Lind P, Oyen WJ, et al.; European Association of Nuclear Medicine (EANM). Guidelines for radioiodine therapy of differentiated thyroid cancer. Eur J Nucl Med Mol Imaging. 2008 Oct;35(10): 1941-59.

23 Silberstein EB, Alavi A, Balon HR, Clarke SE, Divgi C, Gelfand MJ, et al. The SNMMI practice guideline for therapy of thyroid disease with 131I 3.0. J Nucl Med. 2012 Oct;53(10): 1633-51.

24 Perros P, Boelaert K, Colley S, Evans C, Evans RM, Gerrard Ba G, et al.; British Thyroid Association. Guidelines for the management of thyroid cancer. Clin Endocrinol (Oxf). 2014 Jul;81 Suppl 1:1-122. 
25 Schlumberger M, Nutting C, Jarzab B, Elisei R, Siena S, Bastholt R, et al. Exploratory analysis of outcomes for patients with locally advanced or metastatic radioactive iodine-refractory differentiated thyroid cancer (RAIRDTC) receiving open-label sorafenib postprogression on the phase III DECISION trial. Presented at European Thyroid Congress 2014; 2014 Sept 6-10; Santiago de Compostela. Abstract OP87.

26 Brose MS, Frenette CT, Keefe SM, Stein SM. Management of sorafenib-related adverse events: a clinician's perspective. Semin Oncol. 2014 Feb;41 Suppl 2:S1-16.

27 Cabanillas M, Terris DJ, Sabra M; for the American Thyroid Associatio. Information for Clinicians: approach to the patient with progressive radioactive iodine refractory thyroid cancer- When to use systemic therapy. Thyroid. 2017 Jun;27(8):987-93.

28 Van Nostrand D. Radioiodine Refractory Differentiated Thyroid Cancer: Time to Update the Classifications. Thyroid. 2018 Sep;28(9): 1083-93.

29 Haddad RI, Schlumberger M, Wirth LJ, Sherman EJ, Shah MH, Robinson B, et al. Incidence and timing of common adverse events in Lenvatinib-treated patients from the SELECT trial and their association with survival outcomes. Endocrine. 2017 Apr;56(1):121-8.

30 Tuttle RM, Ahuja S, Avram AM, Bernet VJ, Bourguet P, Daniels GH, et al. Controversies, Consensus, and Collaboration in the Use of 131I Therapy in Differentiated Thyroid Cancer: A Joint Statement from the American Thyroid Association, the European Association of Nuclear Medicine, the Society of $\mathrm{Nu}$ clear Medicine and Molecular Imaging, and the European Thyroid Association. Thyroid. 2019 Apr;29(4):461-70.

31 Kist JW, de Keizer B, van der Vlies M, Brouwers AH, Huysmans DA, van der Zant FM, et al.; THYROPET Study Group; other members of the THYROPET Study group are John M.H. de Klerk. 124I PET/CT to Predict the Outcome of Blind 131I Treatment in Patients with Biochemical Recurrence of Differentiated Thyroid Cancer: Results of a Multicenter Diagnostic Cohort Study (THYROPET). J Nucl Med. 2016 May;57(5):701-7.

32 Sabra MM, Grewal RK, Tala H, Larson SM, Tuttle RM. Clinical outcomes following empiric radioiodine therapy in patients with structurally identifiable metastatic follicular cell-derived thyroid carcinoma with negative diagnostic but positive post-therapy 131I whole-body scans. Thyroid. 2012 Sep;22(9): 877-83.

33 Leboulleux S, El Bez I, Borget I, Elleuch M, Déandreis D, Al Ghuzlan A, et al. Postradioiodine treatment whole-body scan in the era of 18-fluorodeoxyglucose positron emission tomography for differentiated thyroid carcinoma with elevated serum thyroglobulin levels. Thyroid. 2012 Aug;22(8):832-8.
34 Schwartz LH, Seymour L, Litière S, Ford R, Gwyther S, Mandrekar S, et al. RECIST 1.1 Standardisation and disease-specific adaptations: Perspectives from the RECIST Working Group. Eur J Cancer. 2016 Jul;62:138-45.

35 Melo M, da Rocha AG, Vinagre J, Batista R, Peixoto J, Tavares C, et al. TERT promoter mutations are a major indicator of poor outcome in differentiated thyroid carcinomas. J Clin Endocrinol Metab. 2014 May;99(5): E754-65.

36 Liu R, Bishop J, Zhu G, Zhang T, Ladenson PW, Xing M. Mortality Risk Stratification by Combining BRAF V600E and TERT Promoter Mutations in Papillary Thyroid Cancer: Genetic Duet of BRAF and TERT Promoter Mutations in Thyroid Cancer Mortality. JAMA Oncol. 2017 Feb;3(2):202-8.

37 Fagin JA, Wells SA Jr. Biologic and Clinical Perspectives on Thyroid Cancer. N Engl J Med. 2016 Sep;375(11):1054-67.

38 Sabra MM, Sherman EJ, Tuttle RM. Tumor volume doubling time of pulmonary metastases predicts overall survival and can guide the initiation of multikinase inhibitor therapy in patients with metastatic, follicular cell-derived thyroid carcinoma. Cancer. 2017 Aug; 123(15):2955-64.

39 Sabra MM, Sherman E, Tuttle RM. Prolongation of tumour volume doubling time (midDT) is associated with improvement in disease-specific survival in patients with rapidly progressive radioactive iodine refractory differentiated thyroid cancer selected for molecular targeted therapy. Clin Endocrinol (Oxf). 2019 Apr;90(4):617-22.

40 Robbins RJ, Wan Q, Grewal RK, Reibke R, Gonen M, Strauss HW, et al. Real-time prognosis for metastatic thyroid carcinoma based on 2-[18F]fluoro-2-deoxy-D-glucose-positron emission tomography scanning. J Clin Endocrinol Metab. 2006 Feb;91(2):498-505.

41 Deandreis D, Al Ghuzlan A, Leboulleux S, Lacroix L, Garsi JP, Talbot M, et al. Do histological, immunohistochemical, and metabolic (radioiodine and fluorodeoxyglucose uptakes) patterns of metastatic thyroid cancer correlate with patient outcome? Endocr Relat Cancer. 2011 Jan;18(1):159-69.

42 Miyauchi A, Kudo T, Miya A, Kobayashi K, Ito Y, Takamura Y, et al. Prognostic impact of serum thyroglobulin doubling-time under thyrotropin suppression in patients with papillary thyroid carcinoma who underwent total thyroidectomy. Thyroid. 2011 Jul;21(7):70716.

43 Orloff LA, Wiseman SM, Bernet VJ, Fahey T] 3rd, Shaha AR, Shindo ML, et al. American Thyroid Association Statement on Postoperative Hypoparathyroidism: Diagnosis, Prevention, and Management in Adults. Thyroid. 2018 Jul;28(7):830-41.

44 Hamilton SN, Tran E, Berthelet E, Wu J. The role of external beam radiation therapy in well-differentiated thyroid cancer. Expert Rev Anticancer Ther. 2017 Oct;17(10):905-10.
45 Chen PV, Osborne R, Ahn E, Avitia S, Juillard G. Adjuvant external-beam radiotherapy in patients with high-risk well-differentiated thyroid cancer. Ear Nose Throat J. 2009 Jul; 88(7):E01.

46 So K, Smith RE, Davis SR. Radiotherapy in well-differentiated thyroid cancer: is it underutilized? ANZ J Surg. 2016 Sep;86(9):696700 .

47 Dunne EM, Fraser IM, Liu M. Stereotactic body radiation therapy for lung, spine and oligometastatic disease: current evidence and future directions. Ann Transl Med. 2018 Jul; 6(14):283.

48 Ribechini A, Bottici V, Chella A, Elisei R, Vitti $P$, Pinchera $A$, et al. Interventional bronchoscopy in the treatment of tracheal obstruction secondary to advanced thyroid cancer. J Endocrinol Invest. $2006 \mathrm{Feb}$;29(2):131-5.

49 Kato S, Murakami H, Demura S, Yoshioka K, Yokogawa N, Yonezawa N, et al. Kidney and Thyroid Cancer-Specific Treatment Algorithm for Spinal Metastases: A Validation Study. World Neurosurg. 2019 Feb; 122: e1305-11.

50 Moneke I, Kaifi JT, Kloeser R, Samson P, Haager B, Wiesemann S, et al. Pulmonary metastasectomy for thyroid cancer as salvage therapy for radioactive iodine-refractory metastases. Eur J Cardiothorac Surg. 2018 Mar; 53(3):625-30.

51 Cazzato RL, Garnon J, Koch G, Shaygi B, Tsoumakidou G, Caudrelier J, et al. Current role of interventional radiology in the management of visceral and bone metastases from thyroid cancer. Gland Surg. 2018 Apr;7(2):80-8.

52 Young M, John S: Hepatic Chemoembolization. StatPearls [Internet]. Treasure Island (FL): StatPearls Publishing; 2018 Jan-. 2019 Jan 27.

53 Minocha J, Salem R, Lewandowski RJ. Transarterial chemoembolization and yittrium-90 for liver cancer and other lesions. Clin Liver Dis. 2014 Nov; 18(4):877-90.

54 Choy PY, Koea J, McCall J, Holden A, Osbourne $\mathrm{M}$. The role of radiofrequency ablation in the treatment of primary and metastatic tumours of the liver: initial lessons learned. NZMed J.2002 Aug;115(1159):U128.

55 Mazzeo S, Cervelli R, Elisei R, Tarantini G, Cappelli C, Molinaro E, et al. mRECIST criteria to assess recurrent thyroid carcinoma treatment response after radiofrequency ablation: a prospective study. J Endocrinol Invest. 2018 Dec;41(12):1389-99.

56 Hay ID, Lee RA, Davidge-Pitts C, Reading CC, Charboneau JW. Long-term outcome of ultrasound-guided percutaneous ethanol ablation of selected "recurrent" neck nodal metastases in 25 patients with TNM stages III or IVA papillary thyroid carcinoma previously treated by surgery and 131I therapy. Surgery. 2013 Dec;154(6):1448-54.

57 Deschamps F, de Baere T. Cementoplasty of bone metastases. Diagn Interv Imaging. 2012 Sep;93(9):685-9. 
58 Gianoukakis AG, Dutcus CE, Batty N, Guo M, Baig M. Prolonged duration of response in lenvatinib responders with thyroid cancer. Endocr Relat Cancer. 2018 Jun;25(6):699704.

59 Brose MS, Smit J, Lin CC, Pitoia F, Fellous M, DeSanctis $Y$, et al. Timing of multikinase inhibitor initiation in differentiated thyroid cancer. Endocr Relat Cancer. 2017 May;24(5): $237-42$.

60 Brose MS, Worden FP, Newbold KL, Guo M, Hurria A. Effect of Age on the Efficacy and Safety of Lenvatinib in Radioiodine-Refractory Differentiated Thyroid Cancer in the Phase III SELECT Trial. J Clin Oncol. 2017 Aug; 35(23):2692-9.

61 Mahajan P, Dawrant J, Kheradpour A, Quintanilla NM, Lopez ME, Orth RC, et al. Response to Lenvatinib in Children with Papillary Thyroid Carcinoma. Thyroid. 2018 Nov; 28(11):1450-4.

62 Robinson B, Schlumberger M, Wirth LJ, Dutcus $\mathrm{CE}$, Song J, Taylor $\mathrm{MH}$, et al. Characterization of Tumor Size Changes Over Time From the Phase 3 Study of Lenvatinib in Thyroid Cancer. J Clin Endocrinol Metab. 2016 Nov;101(11):4103-9.

63 Gotink KJ, Verheul HM. Anti-angiogenic tyrosine kinase inhibitors: what is their mechanism of action? Angiogenesis 2010;13:1e14.

64 Weitzman SP, Sherman SI. Novel Drug Treatments of Progressive Radioiodine-Refractory Differentiated Thyroid Cancer. Endocrinol Metab Clin North Am. 2019 Mar;48(1):25368.

65 Dadu R, Devine C, Hernandez M, Waguespack SG, Busaidy NL, Hu MI, et al. Role of salvage targeted therapy in differentiated thyroid cancer patients who failed first-line sorafenib. J Clin Endocrinol Metab. 2014 Jun; 99(6):2086-94.

66 Cabanillas ME, Schlumberger M, Jarzab B, Martins RG, Pacini F, Robinson B, et al. A phase 2 trial of lenvatinib (E7080) in advanced, progressive, radioiodine-refractory, differentiated thyroid cancer: A clinical outcomes and biomarker assessment. Cancer. 2015 Aug;121(16):2749-56.

67 Schneider TC, Abdulrahman RM, Corssmit EP, Morreau H, Smit JW, Kapiteijn E. Longterm analysis of the efficacy and tolerability of sorafenib in advanced radio-iodine refractory differentiated thyroid carcinoma: final results of a phase II trial. Eur J Endocrinol. 2012 Nov; 167(5):643-50

68 Wirth LJ, Tahara M, Robinson B, Francis S, Brose MS, Habra MA, et al. Treatment-emergent hypertension and efficacy in the phase 3 Study of (E7080) lenvatinib in differentiated cancer of the thyroid (SELECT). Cancer. 2018 Jun;124(11):2365-72.
69 Murgia M, Serrano AL, Calabria E, Pallafacchina G, Lomo T, Schiaffino S. Ras is involved in nerve-activity-dependent regulation of muscle genes. Nat Cell Biol. 2000 Mar;2(3): 142-7.

70 Antoun S, Birdsell L, Sawyer MB, Venner P, Escudier B, Baracos VE. Association of skeletal muscle wasting with treatment with sorafenib in patients with advanced renal cell carcinoma: results from a placebo-controlled study. J Clin Oncol. 2010 Feb;28(6):1054-60.

71 Massicotte $\mathrm{MH}$, Borget I, Broutin S, Baracos VE, Leboulleux S, Baudin E, et al. Body composition variation and impact of low skeletal muscle mass in patients with advanced medullary thyroid carcinoma treated with vandetanib: results from a placebo-controlled study. J Clin Endocrinol Metab. 2013 Jun; 98(6):2401-8

72 Colombo C, De Leo S, Di Stefano M, Vannucchi G, Persani L, Fugazzola L. Primary adrenal insufficiency during lenvatinib or vandetanib and improvement of fatigue after cortisone acetate therapy. J Clin Endocrinol Metab. 2019 Mar;104(3):779-84.

73 Zhang ZF, Wang T, Liu LH, Guo HQ. Risks of proteinuria associated with vascular endothelial growth factor receptor tyrosine kinase inhibitors in cancer patients: a systematic review and meta-analysis. PLoS One. 2014 Mar; 9(3):e90135.

74 Abdulrahman RM, Verloop H, Hoftijzer $\mathrm{H}$, Verburg E, Hovens GC, Corssmit EP, et al. Sorafenib-induced hypothyroidism is associated with increased type 3 deiodination. J Clin Endocrinol Metab. 2010 Aug;95(8):3758-62.

75 Verloop H, Smit JW, Dekkers OM. Sorafenib therapy decreases the clearance of thyrotropin. Eur J Endocrinol. 2013 Jan;168(2):163-7.

76 Kamba T, McDonald DM. Mechanisms of ad verse effects of anti-VEGF therapy for cancer. Br J Cancer. 2007 Jun;96(12):1788-95.

77 Qi W, Shen Z, Tang L, Yao Y. Congestive heart failure risk in cancer patients treated with vascular endothelial growth factor tyrosine kinase inhibitors: a systematic review and meta-analysis of 36 clinical trials. Br J Clin Pharmacol. 2014;78(4):748-62.

78 Pitoia F, Schmidt A, Bueno F, Abelleira E, Jerkovich F. Rare complications of multikinase inhibitor treatment. Arch Endocrinol Metab. 2018;62(6):636-40.

79 Dy GK, Adjei AA. Understanding, recognizing, and managing toxicities of targeted anticancer therapies. CA Cancer J Clin. 2013 JulAug;63(4):249-79.
80 Cavalieri S, Cosmai L, Genderini A, Nebuloni $\mathrm{M}$, Tosoni A, Favales F, et al. Lenvatinib-induced renal failure: two first-time case reports and review of literature. Expert Opin Drug Metab Toxicol. 2018 Apr;14(4):379-85.

81 Temel JS, Greer JA, Muzikansky A, Gallagher ER, Admane S, Jackson VA, et al. Early palliative care for patients with metastatic nonsmall-cell lung cancer. N Engl J Med. 2010 Aug;363(8):733-42.

82 Choksi P, Papaleontiou M, Guo C, Worden F, Banerjee M, Haymart M. Skeletal Complications and Mortality in Thyroid Cancer: A Population-Based Study. J Clin Endocrinol Metab. 2017 Apr;102(4):1254-60.

83 Hortobayi GN, Lipton A, Chew HC, Gradishar WJ, Sauter N, Mohanlal R, et al. Efficacy and safety of continued zoledronic acid every 4 weeks versus every 12 weeks in women with bone metastases from breast cancer: results of the OPTIMIZE-2 trial. J Clin Oncol. 2014;32: $5 s$.

84 Rosen LS, Gordon D, Tchekmedyian NS, Yanagihara R, Hirsh V, Krzakowski M, et al. Long-term efficacy and safety of zoledronic acid in the treatment of skeletal metastases in patients with nonsmall cell lung carcinoma and other solid tumors: a randomized, Phase III, double-blind, placebo-controlled trial. Cancer. 2004 Jun;100(12):2613-21.

85 Orita Y, Sugitani I, Toda K, Manabe J, Fujimoto Y. Zoledronic acid in the treatment of bone metastases from differentiated thyroid carcinoma. Thyroid. 2011 Jan;21(1):31-5.

86 Henry D, Vadhan-Raj S, Hirsh V, von Moos R, Hungria V, Costa L, et al. Delaying skeletalrelated events in a randomized phase 3 study of denosumab versus zoledronic acid in patients with advanced cancer: an analysis of data from patients with solid tumors. Support Care Cancer. 2014 Mar;22(3):679-87.

87 Stopeck AT, Lipton A, Body JJ, Steger GG, Tonkin K, de Boer RH, et al. Denosumab compared with zoledronic acid for the treatment of bone metastases in patients with advanced breast cancer: a randomized, doubleblind study. J Clin Oncol. 2010 Dec;28(35): $5132-9$.

88 Rosenthal MS, Angelos P, Cooper DS, Fassler C, Finder SG, Hays MT, et al.; American Thyroid Association Ethics Advisory Committee. Clinical and professional ethics guidelines for the practice of thyroidology. Thyroid. 2013 Oct;23(10):1203-10.

89 Tufano RP, Noureldine SI, Angelos P. Incidental thyroid nodules and thyroid cancer: considerations before determining management. JAMA Otolaryngol Head Neck Surg. 2015 Jun;141(6):566-72. 